\title{
Numerical Calculation of Stress Intensity Factors for Semi-Elliptical Surface Cracks in Buried-Arc Welded Thick Plates
}

\author{
Krešimir Jukić ${ }^{1}$, Mato Perić ${ }^{2, * \mathbb{D}}$, Zdenko Tonković ${ }^{1}$, Ivica Skozrit ${ }^{1}$ and Tomislav Jarak ${ }^{1}{ }^{\mathbb{D}}$ \\ 1 Faculty of Mechanical Engineering and Naval Architecture, University of Zagreb, Ivana Lučića 5, \\ 10000 Zagreb, Croatia; kresimir.jukic@fsb.hr (K.J.); zdenko.tonkovic@fsb.hr (Z.T.); ivica.skozrit@fsb.hr (I.S.); \\ tomislav.jarak@fsb.hr (T.J.) \\ 2 Bestprojekt d.o.o., Bureau of Energetic and Mechanical Engineering Ltd., Petrovaradinska 7, \\ 10000 Zagreb, Croatia \\ * Correspondence: mato.peric@fsb.hr; Tel.: +385-1-3845-147
}

Citation: Jukić, K.; Perić, M.

Tonković, Z.; Skozrit, I.; Jarak, T. Numerical Calculation of Stress Intensity Factors for Semi-Elliptical Surface Cracks in Buried-Arc Welded Thick Plates. Metals 2021, 11, 1809. https://doi.org/10.3390/met11111809

Academic Editor: Tomasz Kik

Received: 15 October 2021

Accepted: 5 November 2021

Published: 10 November 2021

Publisher's Note: MDPI stays neutral with regard to jurisdictional claims in published maps and institutional affiliations.

Copyright: (C) 2021 by the authors Licensee MDPI, Basel, Switzerland. This article is an open access article distributed under the terms and conditions of the Creative Commons Attribution (CC BY) license (https:// creativecommons.org/licenses/by/ $4.0 /)$
Abstract: The present study deals with the influence of residual stresses induced by the buried-arc welding on the crack behavior in two butt-welded $20 \mathrm{~mm}$ thick plates. The following steps were undertaken: the thermo-mechanical simulation of the welding process, the mapping of stress results from a finite element (FE) mesh used for the welding simulation to a new FE mesh with a crack, the stress balancing, and the stress intensity factor (SIF) calculation. The FE and weight function (WF) methods were used to investigate the SIFs at the deepest point of semi-elliptical surface cracks with different geometries, orientations, and positions in relation to the weld line. In the case of cracks perpendicular to the weld line, the FE and WF results showed a good agreement for smaller cracks, while deviation between the results increases with the size of the crack. Considering the SIF solutions for the cracks of arbitrary orientation, it was observed that for some cases, the SIF value for mode III of crack opening can be of significant influence.

Keywords: buried arc welding; butt-welded plates; stress intensity factor; welding residual stress; semi-elliptical surface crack; finite element analysis; weight function method

\section{Introduction}

The joining of structural components in many industries is usually done by cheap, very reliable, and productive welding technology. This process introduces a large amount of heat into a structure, which leads to the melting of a material in a weld and its surrounding area. Steep cooling gradients after welding lead to the shrinkage of the material in the weld zone in relation to the material away from the weld, which inevitably causes permanent structure deformations and residual stresses [1-7]. The residual stresses, especially in combination with workloads, can induce the initiation of a fatigue crack or cause the brittle fracture or corrosion cracking. Such degradations of materials reduce the service life and the integrity of the structure. For these reasons, it is necessary to monitor the propagation of the crack during the exploitation of the structure [8-11]. Nowadays, due to the rapid development of computer technology, the Finite Element Method (FEM) is used to predict the dynamics of crack propagation, which reduces financial expenses. To calculate the crack growth, it is necessary to know the stress intensity factor (SIF), which for simpler cases can be obtained from industrial codes (API 579-1, BS 7910, ASME FFS-1, etc.) as an analytical solution based on the influence coefficients. A prerequisite for using these codes is the knowledge of the residual stress profile through the thickness of the structure. This profile can be obtained either experimentally (neutron diffraction method [12], contour method [13], etc.) or by using an appropriate numerical method, such as FEM [14,15]. For structures of more complex geometry, SIFs are calculated entirely by numerical procedures, where it is necessary to perform a numerical welding simulation in advance in order to obtain the distribution of residual stresses over the entire welded structure. 
Oh et al. [16] used the example of a semi-elliptical crack in a thick plate to compare the solutions obtained by a numerical procedure with the solutions obtained from engineering codes. They highlighted that order of the fitting curve for SIFs can influence the final solution significantly, and that even the choice of fourth-order polynomial, which is usually recommended, may lead to inaccurate results. Qiang et al. [17] numerically determined the residual stress distribution in a multi-pass welded steel plate. Afterwards, they numerically calculated the SIFs and compared them with the universal weight function (WF) solutions, confirming a good correlation of the results. Lee et al. [18] computed SIFs under the action of residual stresses combined with an external load in a model composed of a butt-weld and a T-joint weld. Three cases were analyzed: a model without residual stresses subjected to a tensile load, a model subjected only to the residual stresses, and a model subjected to a combination of both the residual stresses and the additional tensile load. Then, the models were compared with analytical WF solutions, and a good agreement of the results was found. Gadallah et al. [19] presented a method for evaluating SIFs based on the insertion of crack blocks into a welded model. They studied the effect of crack block mesh density on the value of SIFs for a semi-elliptical crack occurrence in the welded structure. The influence of various heat inputs and residual stresses on the SIF values and the propagation of fatigue crack was investigated by Gadallah et al. [20] in a welded structure containing a semi-elliptical crack. Labeas and Diamantakos [21] calculated the residual stresses in a welded aircraft structure by developing an interpolation technique for transferring the residual stresses from a coarser mesh without any crack to a model with a finer mesh containing a crack, thus obtaining more accurate SIFs.

It is worth noting that all the above papers deal with the computation of SIFs in the presence of residual stresses, optionally combined with an external load. However, only specific crack orientations are considered; namely, the crack is either perpendicular to the welding line or parallel to it. In the present study, the research of SIFs was extended to cracks with arbitrary orientation in relation to the welding direction in thick steel plates that are butt-welded by buried-arc technology.

Many key structural elements of various engineering structures in mechanical engineering, power plants, shipbuilding, etc., are produced as butt-welded metallic plates. The ever-increasing market demands lead to the necessity for a faster production of welded structures, and this in turn motivates the development and introduction of new efficient welding technologies, such as the buried-arc welding. This is a Metal Active Gas (MAG) welding process that uses high electric currents and voltages, which enables the deep penetration of a melting wire tip under the surface of a molten weld pool. This produces less spatter and high welding speeds in comparison to the processes that use open arcs. Consequently, the number of welding passes is reduced, and the overall speed of the welding process is higher when compared to other conventional MAG processes. All this makes the buried-arc welding a very attractive tool in the welding of thick plates. However, since the buried-arc transfer is a relatively new technology in welding, the analyses of the residual stress, thermal, and strain fields, as well as their influence on the welded structures, are still very limited in the scientific and professional literature.

Previously [22-24], the authors of this contribution had studied the distributions of temperature field during the buried-arc welding process, as well as the resulting residual deformation and stresses, in the plates joined by butt or tee welds and in plates containing circular patch samples. This contribution is a natural continuation of the mentioned previous research, and its goal is to investigate the influence of residual stresses produced by the buried-arc welding on the crack behavior in the butt-welded thick plates. For this purpose, in this preliminary step, the variation of the SIF values is investigated, depending on the geometry, orientation, and position of the crack in relation to the weld line.

The paper is organized in five sections, as follows. The description of the SIF calculations, resulting from the residual stresses, is presented in Section 2. In Section 3, the numerical results for the residual stresses in two butt-joint steel plates, induced by the buried-arc welding, are provided and compared to the values obtained by experimental 
measurements. Here, the important details of the applied numerical model are presented, and the performed numerical simulation, consisting of the heat transfer analysis during welding and the subsequent structural analysis, is shortly described. In Section 4, the Finite Element (FE) fracture analyses are conducted to investigate the effect of the obtained stress distribution on SIFs of semi-elliptical surface cracks with different geometries, angular orientations, and positions. The numerical results are compared with those obtained by the WF method. A thorough discussion on the obtained results is given. Finally, concluding remarks and further research plans are drawn in Section 5.

\section{Stress Intensity Factor Determination Due to Residual Stresses}

In the herein presented study, SIFs are calculated by the WF method, which can be applied for arbitrary stress distributions. The expression for SIF calculations has the following form:

$$
K=\int_{0}^{a} w(\xi, a) \sigma(\xi) \mathrm{d} \xi,
$$

where $w(\xi, a)$ is a weight function of the crack, whose value depends on the position coordinate $\xi$ and crack length $a$ (Figures 3 and 4). In Equation (1), $\sigma(\xi)$ denotes the residual stress distribution in the uncracked structure. Here, the residual stress field is approximated as a fourth-order polynomial in terms of $\xi$. For the deepest point of a semi-elliptical crack in a flat plate, which was considered in this work, Shen et al. [25] proposed general weight functions to calculate SIF. The function $w(\xi, a)$ for the deepest point of a semi-elliptical crack is expressed as

$$
w(\xi)=\frac{2}{\sqrt{\pi(a-\xi)}}\left(1+M_{1}\left(1-\frac{\xi}{a}\right)^{\frac{1}{2}}+M_{2}\left(1-\frac{\xi}{a}\right)+M_{3}\left(1-\frac{\xi}{a}\right)^{\frac{3}{2}}\right)
$$

where the parameters $M_{1}, M_{2}$, and $M_{3}$ depend on the geometry of a cracked body and crack size. The expressions for these parameters can be found in various engineering handbooks and technical standards.

In this work, the SIF values were calculated numerically using a built-in J-integral solver integrated in the Abaqus software and the results were compared with those obtained by the above-described WF method. The values of the $J$-integral for the deepest point of a semi-elliptical crack are extracted from the Abaqus and afterwards converted to the SIF value. In the case of welding residual stresses, the semi-elliptical crack is subjected to a mixed crack tip stress mode. In that case, the $J$-integral is expressed as

$$
J=\frac{\left(K_{\mathrm{I}}^{2}+K_{\mathrm{II}}^{2}\right)}{E^{*}}+\frac{K_{\mathrm{III}}^{2}}{2 G}
$$

where $K_{\mathrm{I}}, K_{\mathrm{II}}$, and $K_{\mathrm{III}}$ are the stress intensity factors of mode I (opening mode), mode II (sliding mode), and mode III (tearing mode), respectively. In the above equation, $E^{*}$ is equal to Young's modulus $E$ for plane-stress and $E^{*}=E /\left(1-v^{2}\right)$ for plane-strain and three-dimensional conditions. Here, $v$ is the Poisson's ratio, and $G$ is the shear modulus. To calculate SIF from Equation (3) using an interaction integral method [26], it is necessary to define the auxiliary $J$-integrals as

$$
J_{\mathrm{I}, \text { aux }}=\frac{K_{\mathrm{I}, \text { aux }}^{2}}{E^{*}}, J_{\mathrm{II}, \text { aux }}=\frac{K_{\mathrm{II}, \text { aux }}^{2}}{E^{*}}, J_{\mathrm{III}, \text { aux }}=\frac{K_{\mathrm{III}, \text { aux }}^{2}}{2 G},
$$

which are the product of auxiliary analytical stress fields for modes I, II, and III, respectively. Then, the total $J$-integral is formulated by superimposing the actual and auxiliary stress fields and computing the corresponding $J$-integral $J_{\text {tot }}$. When calculating $K_{\mathrm{I}}$, the auxiliary stress field corresponding to $J_{\mathrm{I} \text {, aux }}$ is superimposed on the actual stress field, leading to 


$$
J_{\text {tot }}=\frac{\left(K_{\mathrm{I}}+K_{\mathrm{I}, \text { aux }}\right)^{2}}{E^{*}}+\frac{K_{\mathrm{II}}^{2}}{E^{*}}+\frac{K_{\mathrm{II}}^{2}}{2 G} .
$$

$K_{\mathrm{I}}$ can be computed by some simple manipulation of Equations (3)-(5) as

$$
K_{\mathrm{I}}=\frac{\left(J_{\text {tot }}-J-J_{\mathrm{I}, \text { aux }}\right) E^{*}}{2 K_{\mathrm{I}, \text { aux }}} .
$$

The SIF values $K_{\text {II }}$ and $K_{\text {III }}$ are calculated analogously by adding auxiliary fields for mode II and III, respectively.

\section{Simulation of Welding Residual Stress}

\subsection{Welded Plates}

Two steel plates made of the S355 J2 $+\mathrm{N}$ material and with dimensions of $300 \mathrm{~mm} \times 300 \mathrm{~mm} \times 20 \mathrm{~mm}$ were butt-welded in a single pass by using the buried-arc welding technology. The plates were welded free, but in the structural analysis, the mechanical constraints were added to prevent the motion of the plates as a rigid body. Applied mechanical constraints are shown in Figure 1. The welding gap between the plates was $3 \mathrm{~mm}$, and the plates were joined using a seven-axis robot. For a better arc stabilization and a higher-quality end configuration, weld-in and weld-out plates were added. The main welding parameters are given in Table 1.

Table 1. Main welding parameters.

\begin{tabular}{cccccccc}
\hline $\begin{array}{c}\text { Welding } \\
\text { Current }\end{array}$ & $\begin{array}{c}\text { Welding } \\
\text { Voltage }\end{array}$ & $\begin{array}{c}\text { Heat Input } \\
\text { Efficiency }\end{array}$ & $\begin{array}{c}\text { Welding } \\
\text { Speed }\end{array}$ & $\begin{array}{c}\text { Wire } \\
\text { Diameter }\end{array}$ & $\begin{array}{c}\text { Wire } \\
\text { Feed Speed }\end{array}$ & $\begin{array}{c}\text { Shielding Gas } \\
\text { Composition }\end{array}$ & $\begin{array}{c}\text { Shielding } \\
\text { Gas Flow }\end{array}$ \\
\hline $540 \mathrm{~A}$ & $41 \mathrm{~V}$ & 0.85 & $300 \mathrm{~mm} / \mathrm{s}$ & $1.6 \mathrm{~mm}$ & $12.3 \mathrm{~m} / \mathrm{min}$ & $100 \% \mathrm{CO}_{2}$ & $25 \mathrm{~L} / \mathrm{min}$ \\
\hline
\end{tabular}

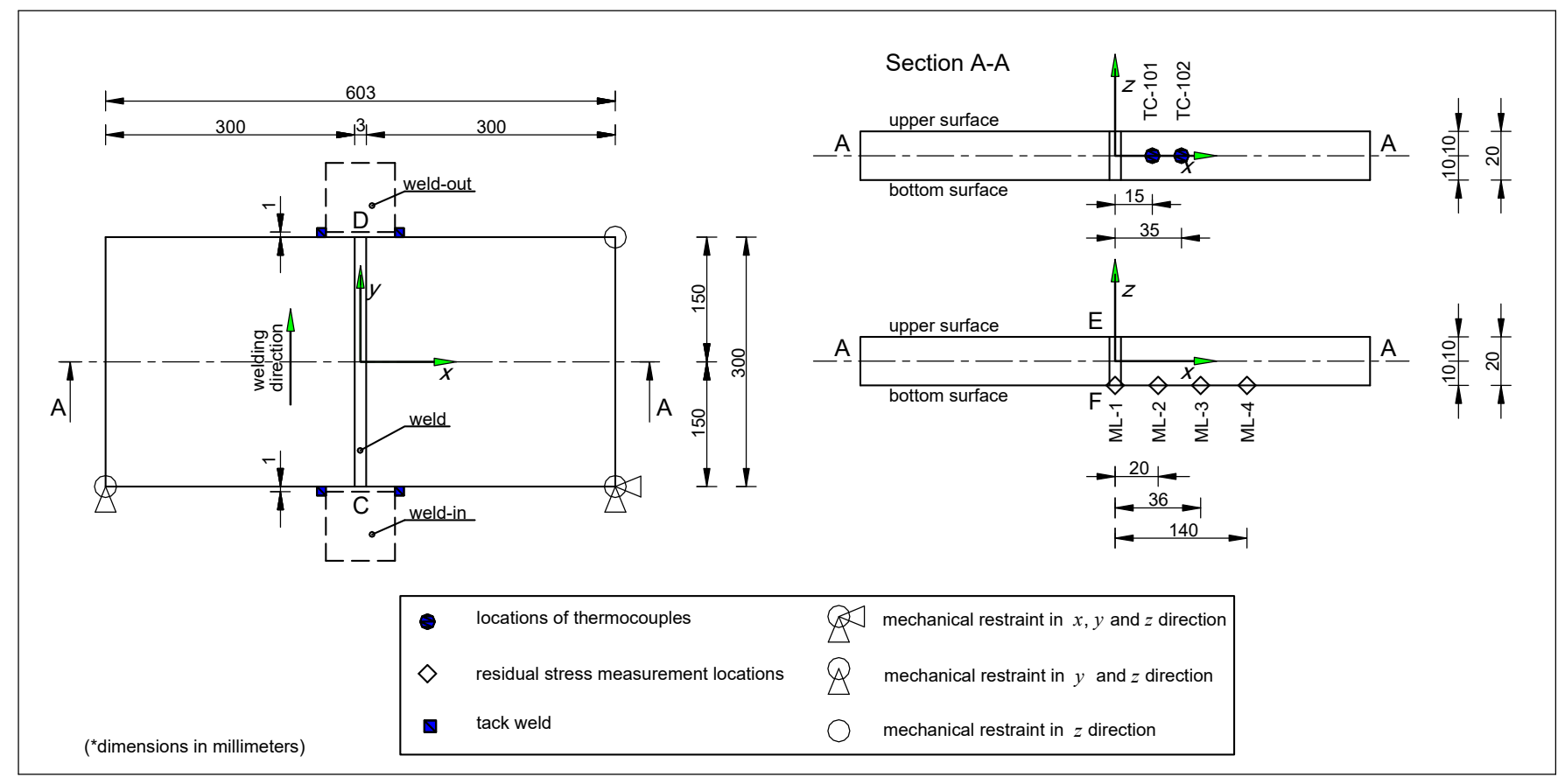

Figure 1. Geometry of the welded model. 
On the middle surface of a plate, two K-type thermocouples (TC-101 and TC-102, were installed, which recorded the history of temperatures during the welding and cooling process to ambient temperature. The thermocouples were placed at $15 \mathrm{~mm}$ and $35 \mathrm{~mm}$ from the weld centerline, respectively. The residual stresses were measured using the hole-drilling strain gauge method on the bottom surface of the plate at four measurement locations (ML-1, ML-2, ML-3, and ML-4). The measurement locations of temperatures and residual stresses, as well as the overall geometry of the welded plate models, are given in Figure 1. A more detailed description of the experimental and numerical procedures used in this research is given in the authors' previous study [22].

\subsection{Heat Transfer Analysis}

A sequential two-step method was applied in the numerical simulation of the welding process. In the first step, a heat transfer analysis was performed to obtain the spatiotemporal distribution of temperatures from the beginning of the welding process to the end of the cooling stage to the ambient temperature. A moving heat source with the uniform distribution $Q=6.27 \times 10^{10} \mathrm{Jm}^{-3} \mathrm{~s}^{-1}$ over the weld elements was applied. The heat convection and radiation boundary conditions were assumed on the outer surfaces of the welded plates with the following properties: the heat transfer coefficient $h_{c}=10 \mathrm{Wm}^{-2} \mathrm{~K}^{-1}$ and the emissivity $\varepsilon=0.9$. For this purpose, in the numerical simulation, the total weld volume was divided into 60 blocks made up of sets of finite elements. Here, the element birth and death technique was employed in the Abaqus to simulate the filler metal deposition. After removal (element death), each element was first reactivated (element birth), and then, the heat source $Q$ was added, simulating the moving of the welding torch. The entire simulation consisted of 60 steps, and in each step, one block was added. After adding the last block, the cooling process in a duration of $7500 \mathrm{~s}$ was started.

\subsection{Structural Analysis}

The obtained temperature distribution in the heat transfer analysis was applied as the thermal load in the numerical structural analysis. In order to reduce the time duration, the structural analysis was conducted within only one step [23]. This analysis was performed by using the same finite element mesh as in the heat transfer analysis, consisting of 36,960 finite elements. However, the DC3D8 elements used in the heat transfer analysis were converted to the C3D8I finite elements in the structural analysis. All the thermal and mechanical properties of the materials were taken as temperature dependent, and more detailed data can be found in [24]. The base material and the weld filler material were modeled as elastic-perfectly plastic [27], and the von Mises criterion and the associated flow rule were adopted [28-30]. Due to the reasons discussed in [31], neither the influence of the steel phase transformation nor the creep behavior of the material were considered in this work. The sensitivity analyses of the FE mesh in both the heat transfer analysis and structural analysis were performed by a submodeling technique in the weld zone and the heat-affected zone, where the temperature gradients are very high [32]. For a more detailed description of the performed numerical simulations, we refer the reader to the earlier work of the authors [33]. The obtained longitudinal (in the welding direction) and transversal (perpendicular to the welding direction) residual stresses across the thickness of the weld (line E-F) are given in Figure 2a,b. The line E-F is shown in Figure 3. The transversal stress at the distance of $22 \mathrm{~mm}$ from the weld line (line $E^{\prime}-F^{\prime}$ ) is given in Figure 2c. The line $E^{\prime}-F^{\prime}$ is shown in Figure 4. 


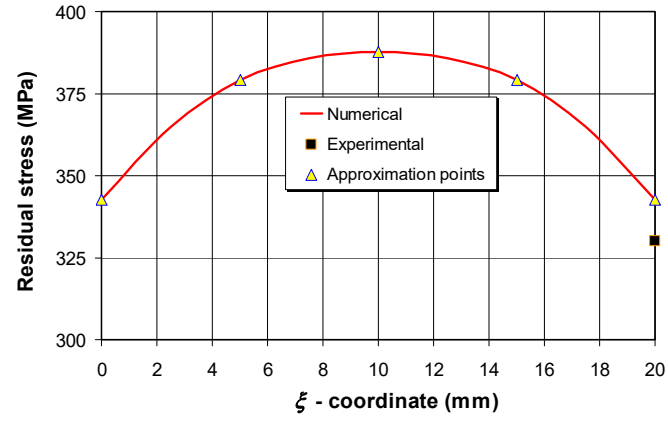

(a)

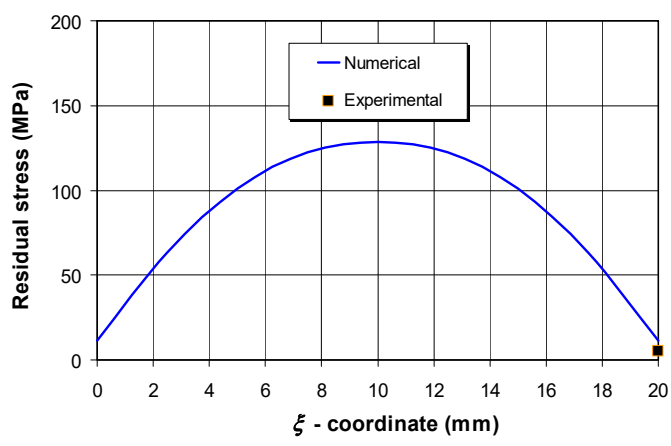

(b)

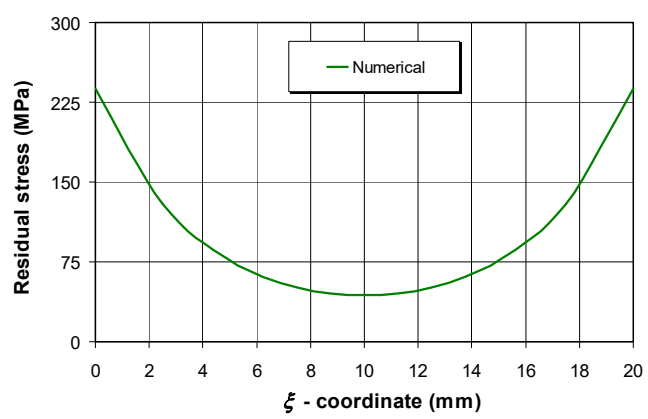

(c)

Figure 2. Through-thickness residual stress profiles in the center of the plate: (a) longitudinal stress, (b) transversal stress, (c) transversal stress at the distance of $22 \mathrm{~mm}$ from the weld line.

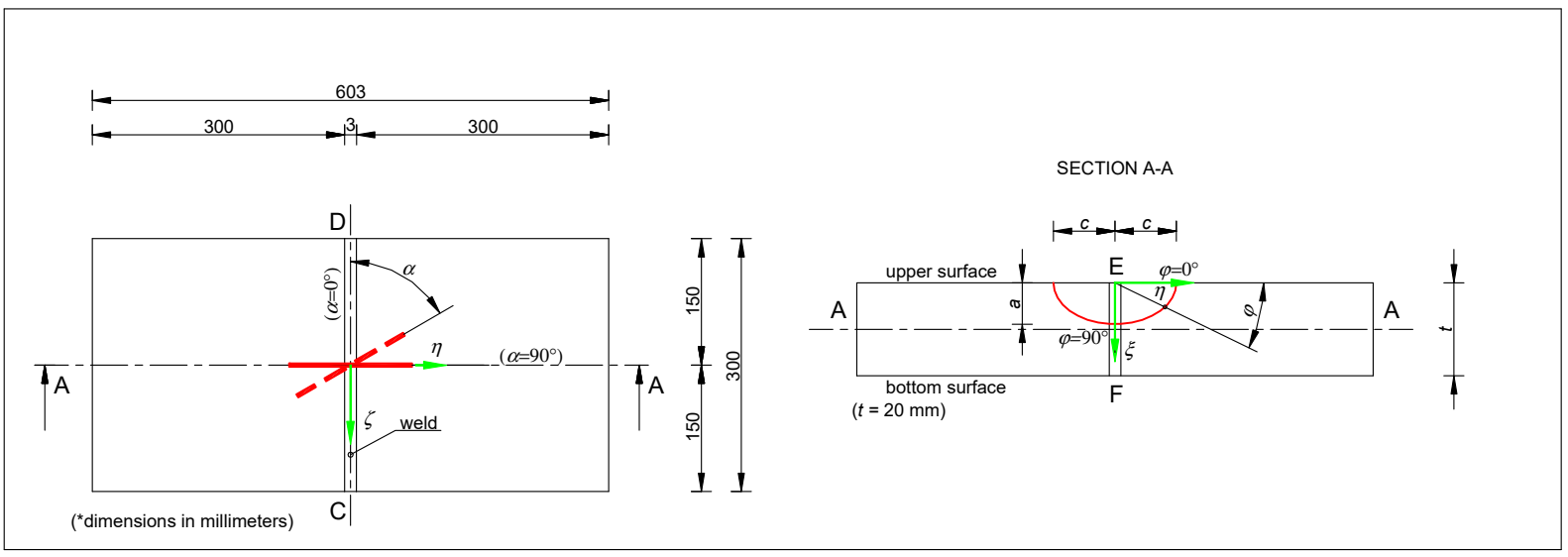

Figure 3. Illustration of shape, dimensions, location, and orientation of cracks over the weld line.

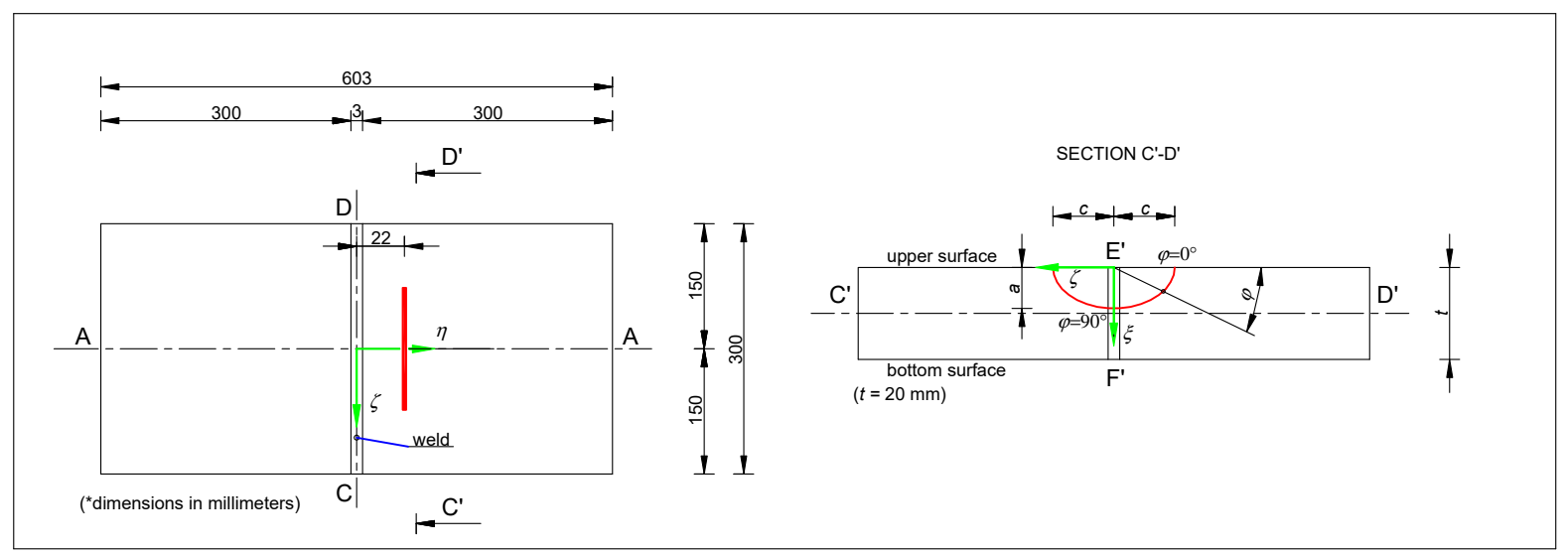

Figure 4. Illustration of shape, dimensions, location, and orientation of cracks parallel to the weld line. 


\section{Effect of Residual Stresses on SIFs-Results and Discussion}

\subsection{Studied Examples}

The previously described welding simulation was followed by fracture mechanics parameter calculations. Since semi-elliptical surface cracks are very common in the engineering practice, this study is limited to the semi-elliptical surface cracks only (Figures 3 and 4) and in the following text, the positions and orientations of the cracks were discussed. In general, the distribution of the welding residual stress field is very complex, and therefore, the fracture mechanics parameters calculations can be complicated, as it is not directly clear which crack positions and orientations will be the critical ones. From the stress distributions (see the next subsection), it is obvious that maximal normal stresses are exhibited close to the weld line and not directly on the weld line. Hence, maximal SIF values are expected at the points with maximal normal stresses. Nevertheless, the tensile residual stress zone is followed by a rapid drop of stresses to negative values in the direction to the weld line (Figure 5a). Therefore, the modeling of large cracks perpendicular to the weld line can lead to compressive stresses on the edges of the crack, which would lead to the closing of the crack and respectively to the reduction of the SIF value. In that case, the weight function (WF) method would overestimate SIF, as it assumes that the stress field changes through the thickness but remains constant in the in-plane directions. Hence, it can be argued that the most conservative predictions of SIF values for all crack sizes would be for the cracks with the center on the weld line (Figure 3). Therefore, four different orientations of cracks at the center of the plate were studied, with $\alpha=90^{\circ}, 60^{\circ}, 30^{\circ}$, and $0^{\circ}$. The parametric study was performed by considering four different crack depth-to-width ratios, $a / c=1$, $0.5,0.25$, and 0.125 , and five different "crack depth"-to-"plate thickness" ratios, including $a / t=0.1,0.2,0.4,0.6$, and 0.8. Herein, $a$ denotes the crack depth, $c$ stands for the crack half-length, and $t$ is the plate thickness, as depicted in Figures 3 and 4. For control, SIFs were also calculated using the WF method from [26], which is contained in all major design codes. For the WF calculations, the through-thickness residual stress distributions in the uncracked plate from Figure 2 were approximated by a fourth-order polynomial through thickness in the form

$$
\sigma(\xi)=\sigma_{0}+\sigma_{1}\left(\frac{\xi}{t}\right)+\sigma_{2}\left(\frac{\xi}{t}\right)^{2}+\sigma_{3}\left(\frac{\xi}{t}\right)^{3}+\sigma_{4}\left(\frac{\xi}{t}\right)^{4} .
$$

The stress coefficients $\sigma_{0}, \sigma_{1}, \sigma_{2}, \sigma_{3}$, and $\sigma_{4}$ are given in Table 2 and were obtained by solving a system of five linear equations with five unknowns. The five selected points at which the through-thickness longitudinal residual stress profile was approximated by a fourth-order polynomial are shown in Figure 2a. Additionally, since the transversal stresses exhibit their highest values at the distance of $22 \mathrm{~mm}$ from the weld line (Figure 5b), at that position, cracks parallel to the weld line (Figure 4) were also studied.

Table 2. Stress coefficients of fourth-order polynomial stress distribution.

\begin{tabular}{cccccc}
\hline $\begin{array}{c}\text { Stress Coefficients } \sigma_{i} \\
\text { for Angle } \alpha\end{array}$ & $\sigma_{0}$ (MPa) & $\sigma_{1}(\mathbf{M P a})$ & $\sigma_{2}$ (MPa) & $\sigma_{3}$ (MPa) & $\sigma_{4}$ (MPa) \\
\hline$\alpha=90^{\circ}$ & 342.6 & 236.0 & -457.7 & 443.3 & -221.7 \\
\hline
\end{tabular}

\subsection{Residual Stress Mapping and Balancing}

As is well known, both the welding simulation and the fracture mechanics simulation require specially designed meshes. During the welding simulation, high temperature and stress gradients can occur near the weld line, and therefore, finer mesh is needed around it. In addition, since there the moving heat source should be defined, a mesh near the weld line should preferably be structured. On the other hand, the fracture mechanics simulations usually require fine-structured meshes around the crack front. Considering all these facts, it is obvious that in practice, it is nearly impossible to design one mesh that would be suitable for both the welding simulation and fracture mechanics simulation, especially 
for cracks not positioned directly on the weld line (Figure 4). Therefore, it is necessary to perform these simulations by using different meshes, which requires a transfer of residual stress solutions from a welding simulation mesh to a fracture mechanics simulation mesh.

There are two relatively obvious ways to obtain a stress field for cracked specimens with the residual stresses calculated for the uncracked specimen, if different meshes are used for each specimen [34]. The first way is to map solutions for the residual stresses from a mesh without a crack to a mesh with a crack, which is followed by the stress balancing. The stress balancing process is necessary, since the introduction of two new crack free surfaces lead to the loss of mechanical equilibrium. Here, the term "opened crack" is used below to indicate that the crack surfaces are free to open in the direction perpendicular to the plane of the crack. In the first step of numerical analysis, the Abaqus command "unbalanced stresses" was used to achieve equilibrium in one increment by using a step variation on the initial condition to resolve the unbalanced stresses. A slightly more complicated procedure is to map the solutions for the residual stresses to a mesh with the crack that is closed by imposing tie kinematic constraints that bound the nodes of two adjacent free surfaces of the crack (here called a "closed crack"). That step is followed by the crack opening, which is simulated by disabling the kinematic constraints. Since the procedure with the stress balancing is simpler, it was used in this work, while the alternative procedure was used for control and verification purposes. It should be noted that although the welding simulation is nonlinear and residual stress values are close to the material yield stress, in both mapping procedures, the material response was assumed to be linear elastic. Since the goal of this research was the calculation of a linear-elastic fracture mechanics (LEFM) parameter, this simplification was justified.

In this subsection, we studied the effect of mapping and stress balancing on the final residual stress distribution. In Figures 5-7, the results of the residual stress mapping are given. The mapping results are shown for the case of a crack perpendicular to the weld and located in the middle of the plate $\left(\alpha=90^{\circ}, a / c=0.25, a / t=0.4\right)$. The distribution of longitudinal residual stresses and transversal residual stresses along the line A-A (Figure 1), calculated on the bottom surface of the plate are presented in Figure 5. It is obvious that the mapping procedure leads to a small deviation with respect to the original residual stress field, which was obtained by using the uncracked mesh. Although this was to be expected due to a coarser cracked mesh and due to the smearing nature of the mapping procedure, generally, a very good agreement was obtained. The only exception is a relatively large deviation of the mapped transversal stress present at the center of the weld line. Having this in mind, it can be predicted that the FEM calculations of SIF for a crack parallel to the weld line will be conservative in comparison to WF calculations, and therefore, the obtained values are acceptable for further analyses.

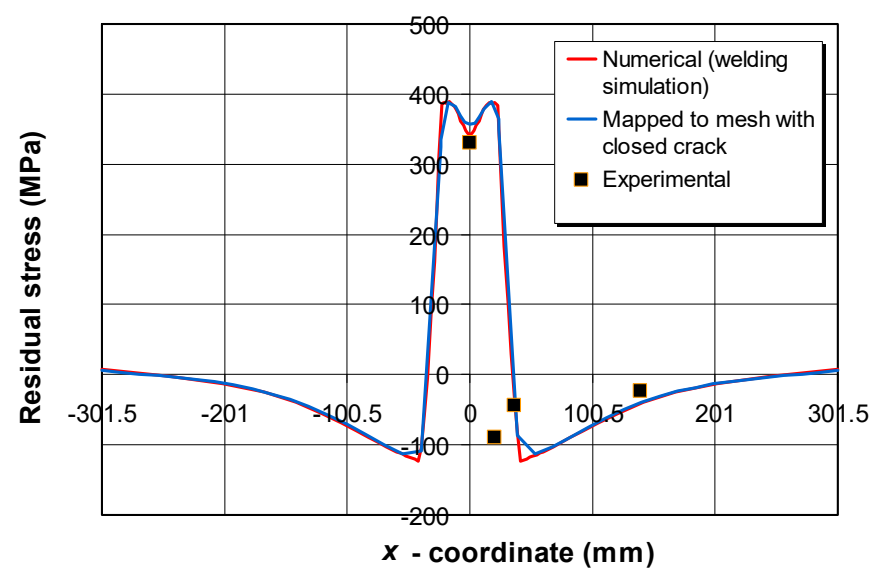

(a)

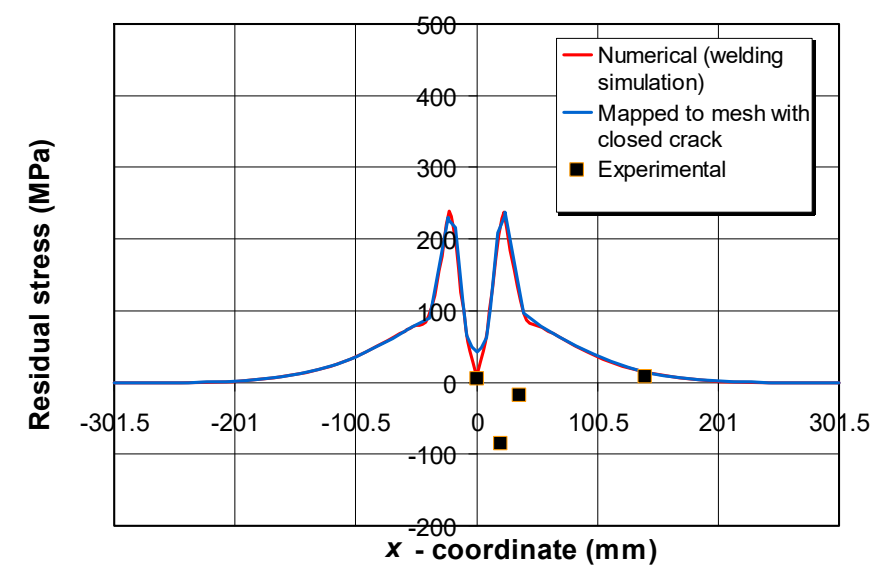

(b)

Figure 5. Residual stress profiles at the bottom surface along line A-A shown in Figure 1 in comparison with experimental measurements: (a) longitudinal stress, (b) transversal stress. 
A more detailed stress field distribution for mapping to the mesh with the closed crack and comparison to the original results can be seen in Figure 6. The longitudinal and transversal residual stresses on the upper surface of the plate are shown, and it is very hard to detect a significant difference between the original results, which was obtained by using the uncracked mesh, and the mapped and balanced values. The tensile longitudinal stresses are a bit higher than the yield stress of the material (355 MPa). Likewise, it is visible that the highest transversal stresses on the bottom plate surface are not positioned directly on the weld line. This can be better observed in Figure 5b. A description of the residual stress measurements and a discussion on the comparison of experimental data with numerical results are given in the authors' previous work [15].

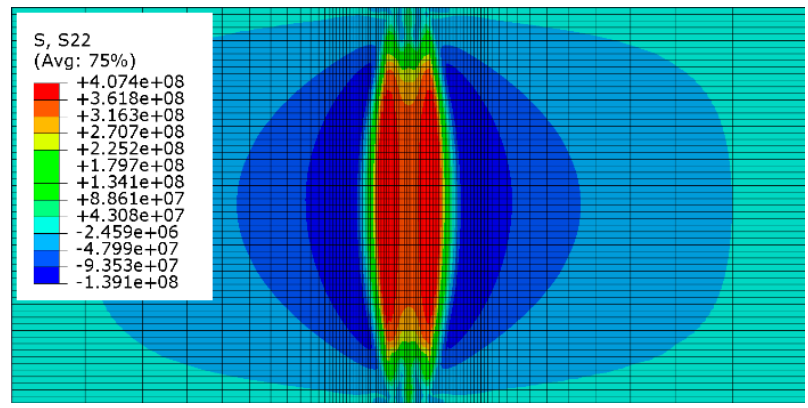

(a)

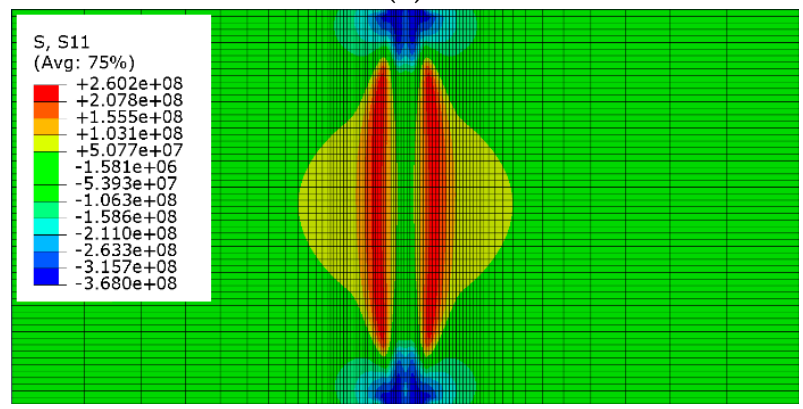

(c)

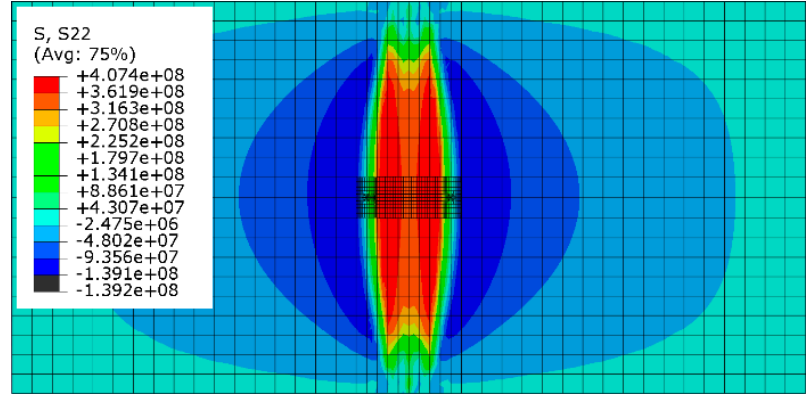

(b)

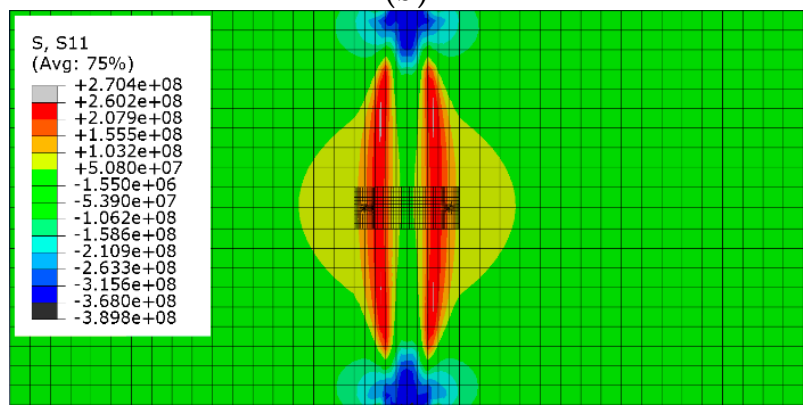

(d)

Figure 6. (a) Longitudinal stress after welding simulation, (b) longitudinal stress after mapping to mesh with closed crack, (c) transversal stress after welding simulation, (d) transversal stress after mapping to mesh with closed crack. All units are in $(\mathrm{Pa})$.

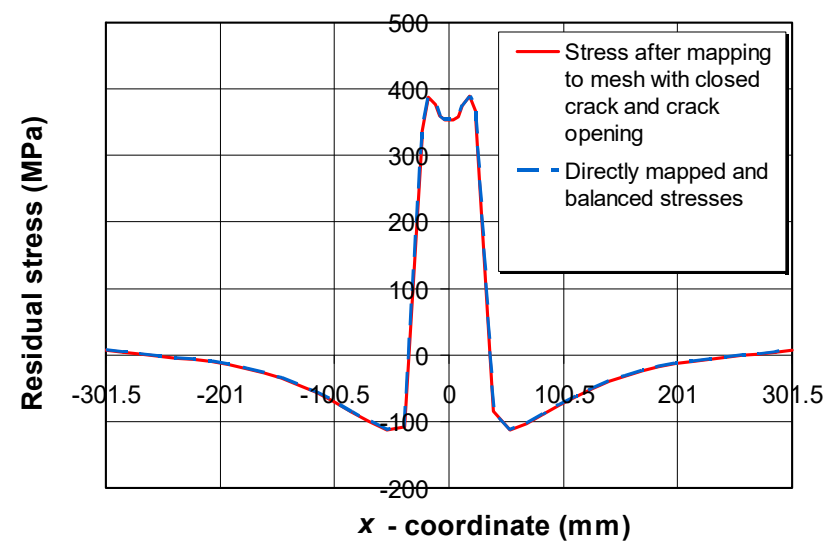

(a)

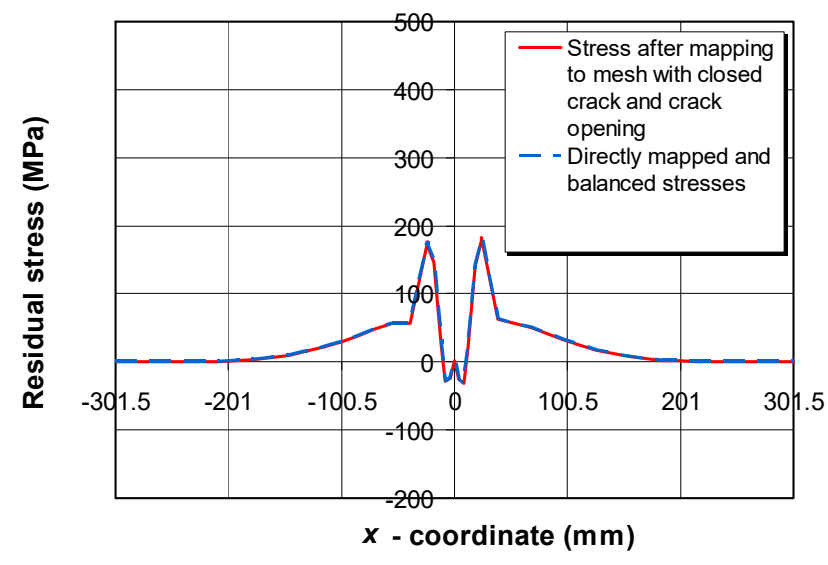

(b)

Figure 7. Residual stresses after stress balancing in a plate with a crack: (a) longitudinal stress, (b) transversal stress. The stresses are computed at the bottom plate surface along line A-A, as shown in Figure 1. 
Furthermore, the effect of the stress-balancing procedure on the final residual stress distribution was studied. The results obtained by both procedures, i.e., the residual stress mapping to the mesh with a closed crack that is subsequently opened and the procedure with the mapping of residual stresses to the mesh with unrestrained crack surfaces followed by the stress balancing, are shown in Figure 7 . Since the results of both procedures are nearly identical, this confirms that our procedure with the stress balancing works correctly.

\subsection{Meshing and Mesh Density Influence}

In this subsection, the influence of mesh density around the crack on the SIF values was performed, and the results are presented. To create meshes with cracks and perform three-dimensional (3D) fracture mechanics simulations, the computer software Zencrack based on FEM was used. Here, the creation of mesh with a crack is done in two steps. In the first step, the uncracked mesh must be prepared. It consists of a group of finite elements near the crack (a near-crack block), which are shown as blue elements, upper left, and a mesh further away from the crack, which surrounds the near-crack block (a far-crack mesh) (Figure 8). The near-crack block consists of a certain number of hexahedral elements that are deleted and replaced with a dense mesh modeling the crack (a cracked block). The uncracked near-crack block has to be organized in the $2 \times n \times 1$ structure; i.e., the block mesh has to be structured and organized in two layers of elements around the crack (one layer for each free surface), where each layer contains $n$ elements and where the crack is positioned between these two layers. In contrast, the cracked blocks consist of dense structured hexahedral meshes. The mesh of a cracked block is connected with the remaining mesh (far-crack mesh) with the tie kinematic constraint. The far-crack mesh can be of arbitrary structure and can consist of arbitrary elements. Since the studied specimen is simple, structured hexahedral meshes have been used in all presented cases. The secondorder elements C3D20 from the Abaqus library have been used. The process of creating a mesh is shown in Figure 8.
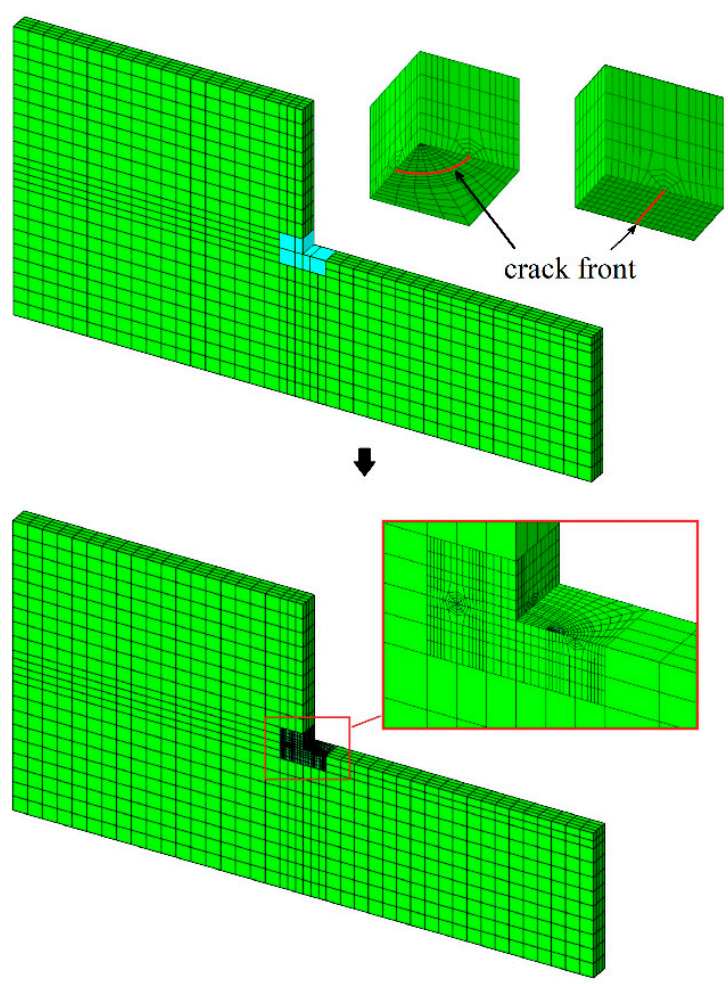

Figure 8. Process of mesh creation.

To ensure that the used meshes are sufficiently dense to solve the problem, we performed the convergence study of SIF values. Meshes of various topologies were considered 
for cracks oriented perpendicularly and at an arbitrary angle relative to the weld line, with different depths of cracked blocks. Three meshes of different densities have been considered. The coarsest mesh ("coarse") consisted of a coarse far-crack mesh and a relatively coarse near-crack mesh. The medium-density mesh ("medium") had the identical nearcrack mesh as the coarsest mesh and a much finer far-crack mesh. The finest mesh ("fine") had the identical far-crack mesh as the medium mesh, while the near-crack mesh was significantly refined. The influence of mesh density on SIF values $\left(K_{\mathrm{I}}\right)$ is shown in Table 3.

Table 3. SIF results for different meshes.

\begin{tabular}{rcccc}
\hline$a / t$ & & & 0.4 & \\
\hline$a / c$ & & 1 & 0.5 & 0.25 \\
\hline \multirow{3}{*}{$K_{\mathrm{I}}\left(\mathrm{MPa} \mathrm{mm}^{1 / 2}\right)$} & coarse & 1302.3 & 1843.0 & 2341.7 \\
\cline { 2 - 5 } & medium & 1305.9 & 1846.0 & 2345.1 \\
\cline { 2 - 5 } & fine & 1302.5 & 1845.6 & 2344.9 \\
\hline
\end{tabular}

As it may be seen, the transition from the "coarse" to the "medium" mesh topology resulted in a very small change in the SIF value, which means that the far-crack mesh is of sufficient density. Similarly, the transition from the "medium" to the "fine" mesh topology resulted in a very small change of the SIF value, and therefore, it is considered that the nearcrack mesh is sufficiently fine. Quantitively, the deviation of results obtained by different mesh topologies was less than $0.2 \%$, which leads to the conclusion that the "coarse" mesh was sufficient to accurately calculate SIF. Such high accuracy obtained by a relatively coarse mesh can be attributed to fact that the second-order elements were used. The non-monotonous convergence of SIF value can be the consequence of the errors caused by the mapping procedure and slightly different element topologies in the region around the crack.

\subsection{SIF Solutions}

In this subsection, the obtained SIFs for all the considered crack configurations are presented. The results obtained by FEM and the Weight Function (WF) method were compared. The through-thickness stresses obtained by Equation (7) were used for the WF calculations. Since it is hard to calculate SIF for a surface point $\left(\varphi=0^{\circ}\right)$ of the crack due to a singularity that differs from the $r^{-0.5}$ singularity, only the results obtained for the deepest point of the crack $\left(\varphi=90^{\circ}\right)$ are shown. In addition, it should be noted that SIF at the deepest point is usually higher than SIF at the surface point. However, this is not a strict rule, and one should be careful when neglecting the surface points SIF values.

To calculate the SIF, the contour integral method in Abaqus was applied, and the obtained results are shown in Figures 9-14. Here, Figures 9-12 present the SIF solutions for cracks at the center of the plate oriented at $\alpha=90^{\circ}, 60^{\circ}, 30^{\circ}$, and $0^{\circ}$ (Figure 3 ), while Figure 13 shows SIF results for cracks with an offset of $\eta=22 \mathrm{~mm}$ and parallel to the weld line $\left(\alpha=0^{\circ}\right)$ (Figure 4$)$. The SIF solutions for the third mode of crack opening are given in Figure 14. The SIF values were computed for a total of three contours around the crack tip. The result from the first contour closest to the crack tip was discarded, and an average SIF was calculated with the remaining two contour values. The difference in the calculated SIFs for the second and third contour was very small, which was an indicator of the accuracy of SIF computation. The WF solutions are shown only for the cracks perpendicular to the weld line $\left(\alpha=90^{\circ}\right.$, Figure 3). It can be noted that the FEM solutions correspond to the WF results well. Thereby, smaller cracks exhibit a better agreement, while deviation between the FEM and WF results increases with the size of the crack, as is the case for the crack with $a / t=0.6$ and $a / c=0.25$ for which the computed SIF results are shown in Figure 9 . The reason for this phenomenon could be the nonuniformity of the stress field in the plate. As the weight functions are derived under the assumption of a stress field that varies only across the plate thickness, a nonuniform residual stress field that varies in all directions could theoretically lead to mistakes in the SIF calculation. Consequently, it is to be noted 
that for small cracks, the influence of the residual stress nonuniformity is very small, but it increases with the increase in the crack size. For example, in Figure 9, it can be seen that for larger cracks (the cracks with larger $a / c$ ), the WF method overestimates SIF in comparison to FEM. That is probably due to the fact that a large crack enters the zone of compressive longitudinal residual stresses (Figure 7), which encloses the crack and reduces the SIF value. For example, for the case of a crack with $a / t=0.6$ and $a / c=0.25$, the crack half-length is $48 \mathrm{~mm}$, meaning that stress in the uncracked plate at the position of the crack edge is negative. In contrast to the WF method, this effect is caught by FEM.

Considering the values of obtained SIFs, it is obvious that even shallow cracks (small value of $a / t$ ) can lead to SIF values that are relatively high in comparison to the fracture toughness of construction steels, ranging from one thousand to several thousand MPa $\mathrm{mm}^{1 / 2}$. The most critical case is a crack perpendicular to the weld line. The transition to a crack parallel to the weld line leads to smaller SIF values, as presented in Figure 9. As expected, wider and deeper cracks exhibit higher SIF values. A notable exception in the presented results are the SIF values for a crack parallel to the weld line, which is positioned at $\eta=22 \mathrm{~mm}$ (Figure 4), as presented in Figure 13. In that case, for small cracks (large crack $a / c$ ratios), the SIF values stay nearly identical with the increase of the crack depth. Such results can be explained with a convex stress profile through the plate thickness at the position of $\eta=22 \mathrm{~mm}$ (Figure 2c), which is in contrast to the concave profiles obtained at the center of the plate (Figure 2a,b).

Furthermore, as can be seen from Figures 12 and 13, the SIF solutions for the cracks parallel to the weld line $\left(\alpha=0^{\circ}\right)$ with the center on the weld line (Figure 12) and with the center in the highest transversal stress region (Figure 13) are almost the same, although the stress distribution at these positions differs considerably at outer plate surfaces, as presented in Figures 5-7. However, as shown in Figure $2 b, c$, the residual stress profiles through the plate thickness at these positions are different, influencing the results. Such results can be explained with the following considerations. As mentioned above, to calculate the SIF, the contour integral approach was used. Here, the contour integral is expanded into a volume integral in three dimensions over a finite domain surrounding the crack. In the presented work, the SIF values were conducted on three contours around the crack tip. It means that the SIF at a reference point on the crack front depends not only on the stress in this point but also on the stress at all other points of the finite domain surrounding the crack. Thus, the presented SIF values depend not only on the residual stress profiles through the plate thickness presented in Figure $2 b, c$ but also on the stresses at the surrounding points. Furthermore, it is to note for shallow cracks that the calculated SIFs are somewhat higher for the cracks with maximum transversal stresses $(\eta=22 \mathrm{~mm})$ compared to the cracks on the weld line (Figure 12). However, this trend is slowly reversed for deeper cracks. This is also in good correlation to the stress through the thickness profiles shown in Figure $2 b, c$, where it is visible that the stresses around the plate middle thickness (interior of the thickness section) are higher at the weld line. In short, it can be concluded that although similar results were obtained, they exhibit the influence of the stress profiles through the thickness.

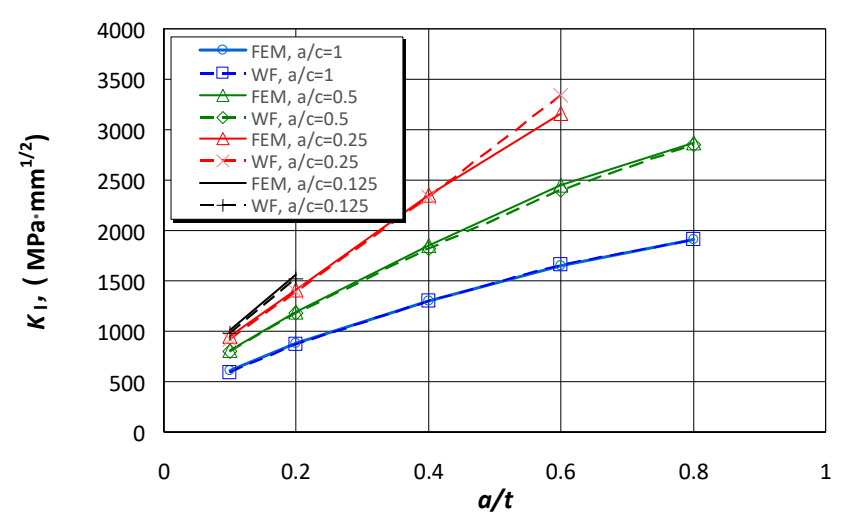

Figure 9. SIF solutions for crack perpendicular to weld line $\left(\alpha=90^{\circ}\right)$. 


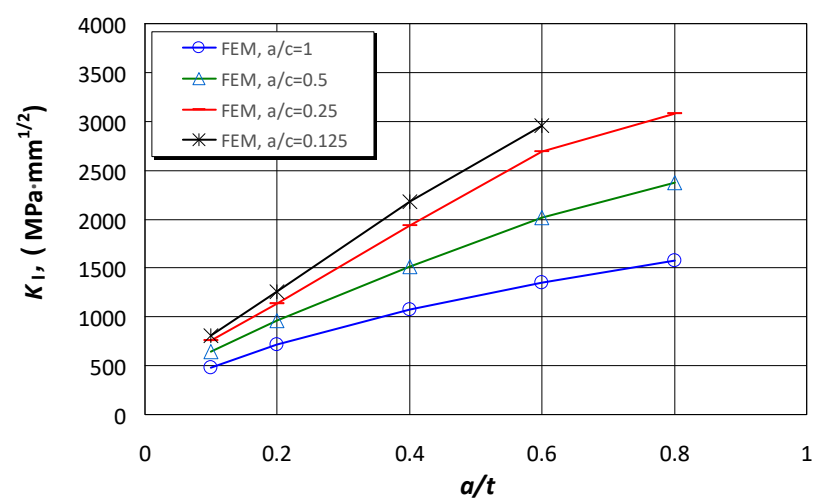

Figure 10. SIF solutions for crack oriented at $\alpha=60^{\circ}$.

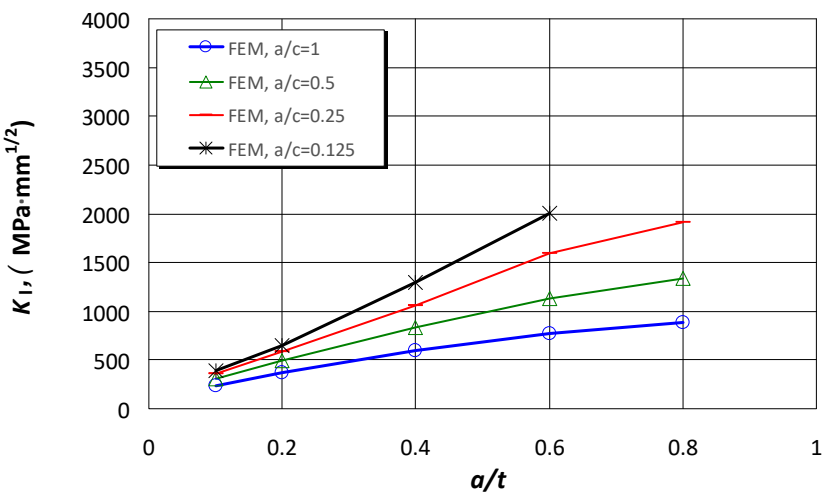

Figure 11. SIF solutions for crack oriented at $\alpha=30^{\circ}$.

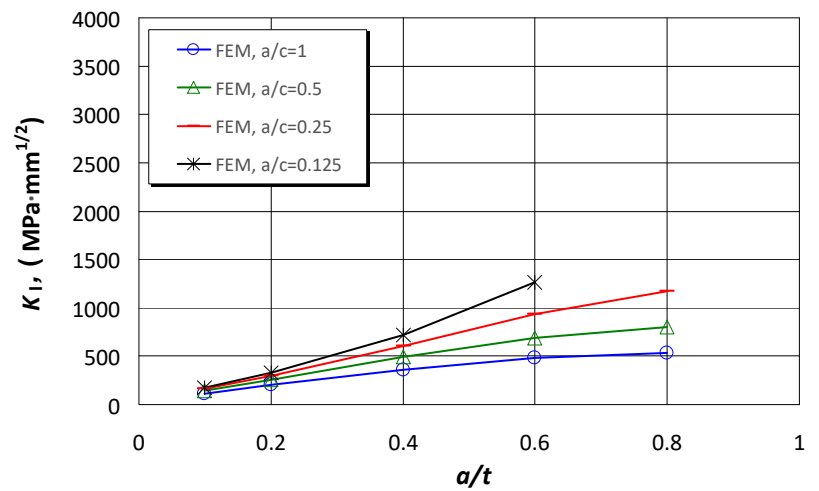

Figure 12. SIF solutions for crack parallel to weld line $\left(\alpha=0^{\circ}\right)$.

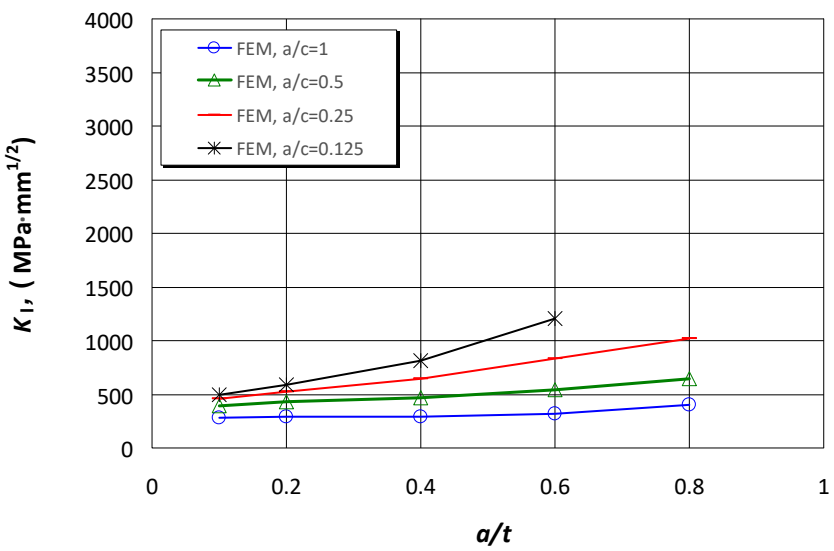

Figure 13. SIF solutions for crack parallel to weld line $\left(\alpha=0^{\circ}\right)$ at $\eta=22 \mathrm{~mm}$ (Figure 4). 


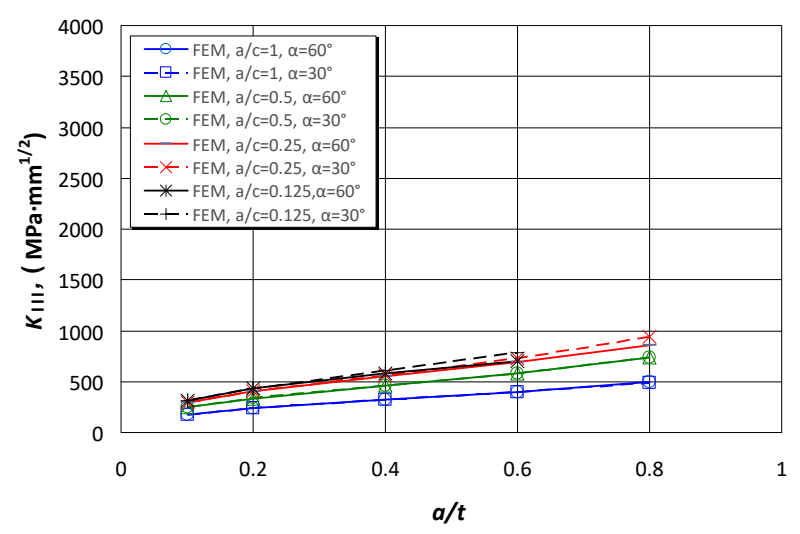

Figure 14. SIF solutions for third mode of crack opening.

Considering the SIF solutions for the deepest point of semi-elliptical surface cracks of arbitrary orientation $\left(0^{\circ}<\alpha<90^{\circ}\right)$, it has been noticed that in some cases, the SIF value for mode III or the out-of-plane shear (tearing) crack mode can have a significant influence (Figure 14). For example, for smaller cracks with $\alpha=30^{\circ}$, the SIF value for mode III is even higher than SIF for mode I. That means that neglecting mode III can lead to the underestimation of effective SIF. As the matter of fact, it seems that in general, this is the case for cracks with $\alpha$ closer to $0^{\circ}$ (cracks that are almost parallel to the weld line). On the other hand, for cracks with $\alpha$ being closer to $90^{\circ}$, e.g., $\alpha=60^{\circ}$, the SIF values for mode I are high, and hence, the influence of the SIF for mode III is less significant. From Figure 14, it can be further noticed that for the crack orientations of $30^{\circ}$ and $60^{\circ}$, for all the considered crack aspect ratios $(a / c)$, the SIF values for the mode III fracture increase with the increase of crack depth ratio $(a / t)$ and with the decrease of crack aspect ratio. This means that the mode III fracture becomes significant at greater crack depths and lengths. In addition, the contribution of stress intensities from mode II can be neglected in comparison with modes I and III.

\section{Conclusions}

The procedure for the determination of LEFM parameters due to welding residual stresses is presented. As a first step, the numerical simulation of the buried-arc welding process for the $20 \mathrm{~mm}$ thick butt-welded plates model in one pass was carried out. Then, a mapping of residual stresses from a mesh for the welding simulation to a new FE mesh used for fracture mechanics analysis was performed. Based on the obtained stress distribution, the FE and WF methods were employed to study its influence on the SIFs at the deepest point of semi-elliptical surface cracks with different geometries, locations, and angular orientations in relation to the weld line. Four different orientations of cracks, with five relative depth ratios and four crack aspect ratios, and with the center on the weld line were studied. Additionally, the cracks parallel to the weld line with the center in the highest transversal stress region were also investigated.

Based on the presented study results, the following conclusions can be summarized:

- The solution mapping and residual stress balancing procedure was verified, and due to a coarser cracked mesh and the smearing nature of the mapping procedure, a small deviation of the mapped and balanced stress distribution with respect to the original residual stress field, obtained by using the uncracked mesh, was observed.

- The FEM-based SIF calculation results were compared to the WF method solutions with a good agreement. Considering the cracks perpendicular to the weld line, it was concluded that the WF method is suitable for small cracks, while in the case of larger cracks, a small error can occur due to the complexity of the residual stress distribution.

- For some cases of cracks of arbitrary angular orientation, it was observed that the SIF solution for mode III of the crack opening can be the same order of magnitude as the 
SIF value for mode I of crack opening and therefore should be taken into account for the estimation of the effective SIF value.

Since the buried-arc welding is a relatively new technology, for better assessment of fracture mechanics parameters due to welding residual stresses, future work should include an investigation of the influence of different parameters such as the welding parameters, heat source model types, plate dimensions, boundary conditions, and constitutive model types.

Author Contributions: Conceptualization, K.J., M.P.; formal analysis, K.J.; writing—original draft preparation, K.J., M.P.; data curation, M.P.; project administration, M.P., Z.T., I.S., T.J.; funding acquisition, Z.T.; supervision, M.P., Z.T., I.S., T.J. All authors have read and agreed to the published version of the manuscript.

Funding: This work has been supported by the European Union through the European Regional Development Fund, Operational Programme "Competitiveness and Cohesion" 2014-2020 of the Republic of Croatia, project "Protection of Structural Integrity in Energy and Transport" (Zacjel, KK.01.1.1.04.0056).

Data Availability Statement: Not applicable.

Conflicts of Interest: The authors declare no conflict of interest.

\section{References}

1. Sepe, R.; Giannella, V.; Greco, A.; De Luca, A. FEM Simulation and Experimental Tests on the SMAW Welding of a Dissimilar T-Joint. Metals 2021, 11, 1016. [CrossRef]

2. Hammad, A.; Nasser-Abdel, Y.; Shama, M. Rational Design of T-Girders via Finite Element Method. J. Mar. Sci. Appl. 2021, 20, 302-316. [CrossRef]

3. Kik, T.; Moravec, J.; Švec, M. Experiments and Numerical Simulations of the Annealing Temperature Influence on the Residual Stresses Level in S700MC Steel Welded Elements. Materials 2020, 13, 5289. [CrossRef] [PubMed]

4. Jin, D.; Hou, C.; Shen, L. Effect of Welding Residual Stress on the Performance of CFST Tubular Joints. J. Constr. Steel Res. 2021, 184, 106827. [CrossRef]

5. Lorza, R.L.; García, R.E.; Martinez, R.F.; Martinez Calvo, M.Á. Using Genetic Algorithms with Multi-Objective Optimization to Adjust Finite Element Models of Welded Joints. Metals 2018, 8, 230. [CrossRef]

6. Kik, T.; Górka, J. Numerical Simulations of Laser and Hybrid S700MC T-Joint Welding. Materials 2019, 12, 516. [CrossRef]

7. Podder, D.; Gupta, O.P.; Das, S.; Mandal, N.R. Experimental and Numerical Investigation of Effect of Welding Sequence on Distortion of Stiffened Panels. Weld. World 2019, 63, 1275-1289. [CrossRef]

8. Sepe, R.; Wiebesiek, J.; Sonsino, C.M. Numerical and Experimental Validation of Residual Stresses of Laser-Welded Joints and Their Influence on the Fatigue Behaviour. Fatigue Fract. Eng. Mater. Struct. 2020, 43, 1126-1141. [CrossRef]

9. Giannella, V.; Fellinger, J.; Perella, M.; Citarella, R. Fatigue Life Assessment in Lateral Support Element of a Magnet for Nuclear Fusion Experiment "Wendelstein 7-X". Eng. Fract. Mech. 2017, 178, 243-257. [CrossRef]

10. Giannella, V. Stochastic Approach to Fatigue Crack-Growth Simulation for a Railway Axle under Input Data Variability. Int. J. Fatigue 2021, 144, 106044. [CrossRef]

11. Keprate, A.; Chandima Ratnayake, R.N.; Sankararaman, S. Experimental Validation of the Adaptive Gaussian Process Regression Model Used for Prediction of Stress Intensity Factor as an Alternative to FEM. J. Offshore Mech. Arct. 2019, 141, 021606. [CrossRef]

12. Jiang, W.; Woo, W.; Wan, Y.; Luo, Y.; Xie, X.; Tu, S.T. Evaluation of Through-Thickness Residual Stresses by Neutron Diffraction and Finite-Element Method in Thick Weld Plates. J. Press. Vessel Technol. ASME 2017, 139, 031401. [CrossRef]

13. Murugan, N.; Narayanan, R. Finite Element Simulation of Residual Stresses and their Measurement by Contour Method. Mater. Des. 2009, 30, 2067-2071. [CrossRef]

14. Seleš, K.; Perić, M.; Tonković, Z. Numerical Simulation of a Welding Process Using a Prescribed Temperature Approach. J. Constr. Steel Res. 2018, 145, 49-57. [CrossRef]

15. Sepe, R.; Armentani, E.; Lamanna, G.; Caputo, F. Evaluation by FEM of the influence of the preheating and post-heating treatments on residual stresses in welding. Key Eng. Mater. 2015, 628, 93-96. [CrossRef]

16. Oh, C.-Y.; Kim, Y.-J.; Oh, Y.-J.; Kim, J.-S.; Song, T.-K.; Kim, Y.-B. Evaluation of Stress Intensity Factors due to Welding Residual Stresses for Circumferential Cracked Pipes. Int. J. Press. Vessel. Pip. 2013, 105-106, 36-48. [CrossRef]

17. Qiang, B.; Li, Y.; Yao, C.; Wang, X. Through-Thickness Welding Residual Stress and its Effect on Stress Intensity Factors for Semi-Elliptical Surface Cracks in a Butt-Welded Steel Plate. Eng. Fract. Mech. 2018, 193, 17-31. [CrossRef]

18. Lee, J.-H.; Jang, B.-S.; Kim, H.-J.; Shim, S.-H.; Im, S.W. The Effect of Weld Residual Stress on Fracture Toughness at the Intersection of Two Welding Lines of Offshore Tubular Structure. Mar. Struct. 2020, 71, 102708. [CrossRef]

19. Gadallah, R.; Osawa, N.; Tanaka, S. Evaluation of Stress Intensity Factor for a Surface Cracked Butt Welded Joint Based on Real Welding Residual Stress. Ocean Eng. 2017, 138, 123-139. [CrossRef] 
20. Gadallah, R.; Osawa, N.; Tanaka, S.; Tsutsumi, S. Critical Investigation on the Influence of Welding Heat Input and Welding Residual Stress on Stress Intensity Factor and Fatigue Crack Propagation. Eng. Fail. Anal. 2018, 89, 200-221. [CrossRef]

21. Labeas, G.; Diamantakos, I. Laser Beam Welding Residual Stresses of Cracked T-joints. Theor. Appl. Fract. Mech. 2013, 63-64, 69-76. [CrossRef]

22. Perić, M.; Garašić, I.; Tonković, Z.; Vuherer, T.; Nižetić, S.; Dedić-Jandrek, H. Numerical Prediction and Experimental Validation of Temperature and Residual Stress Distributions in Buried-Arc Welded Thick Plates. Int. J. Energy Res. 2019, 43, 3590-3600. [CrossRef]

23. Perić, M.; Nižetić, S.; Tonković, Z.; Garašić, I.; Horvat, I.; Boras, I. Numerical Simulation and Experimental Investigation of Temperature and Residual Stress Distributions in a Circular Patch Welded Structure. Energies 2020, 13, 5423. [CrossRef]

24. Perić, M.; Nižetić, S.; Garašić, I.; Gubeljak, N.; Vuherer, T.; Tonković, Z. Numerical Calculation and Experimental Measurement of Temperatures and Welding Residual Stresses in a Thick-Walled T-Joint Structure. J. Therm. Anal. Calorim. 2020, 141, 313-322. [CrossRef]

25. Shen, G.; Plumtree, A.; Glinka, G. Weight Function for the Surface Point of Semi-Elliptical Surface Crack in a Finite Thickness Plate. Eng. Fract. Mech. 1991, 40, 167-176. [CrossRef]

26. Walters, M.C.; Paulino, G.H.; Dodds, R.H., Jr. Interaction Integral Procedures for 3-D Curved Cracks Including Surface Tractions. Eng. Fract. Mech. 2005, 72, 1635-1663. [CrossRef]

27. Gannon, L.; Liu, Y.; Pegg, M.; Smith, M. Effect of Welding Sequence on Residual Stress and Distortion in Flat-Bar Stiffened Plates. Mar. Struct. 2010, 23, 385-404. [CrossRef]

28. Nguyen, K.; Nasouri, R.; Bennett, C.R.; Matamoros, A.; Li, J.; Montoya, A.H. Thermomechanical Modeling of Welding and Galvanizing a Steel Beam Connection Detail to Examine Susceptibility to Cracking. Mater. Perform. Charact. 2018, 7, 165-190. [CrossRef]

29. Perić, M.; Tonković, Z.; Rodić, A.; Surjak, M.; Garašić, I.; Boras, I.; Švaić, S. Numerical Analysis and Experimental Investigation of Welding Residual Stresses and Distortions in a T-Joint Fillet Weld. Mater. Des. 2014, 53, 1052-1063. [CrossRef]

30. Perić, M.; Garašić, I.; Nižetić, S.; Dedić-Jandrek, H. Numerical Analysis of Longitudinal Residual Stresses and Deflections in a T-joint Welded Structure Using a Local Preheating Technique. Energies 2018, 11, 3487. [CrossRef]

31. Deng, D. FEM Prediction of Welding Residual Stress and Distortion in Carbon Steel Considering PhaseTransformation Effects. Mater. Des. 2009, 30, 359-366. [CrossRef]

32. Perić, M.; Tonković, Z.; Maksimović, K.S.; Stamenković, D. Numerical Analysis of Residual Stresses in a T-Joint Fillet Weld Using a Submodeling Technique. FME Trans. 2019, 47, 183-189. [CrossRef]

33. Perić, M.; Seleš, K.; Tonković, Z.; Lovrenić-Jugović, M. Numerical Simulation of Welding Distortions in Large Structures with a Simplified Engineering Approach. Open Phys. 2019, 17, 719-730. [CrossRef]

34. Abaqus, V. Abaqus/Standard User's Manual and Abaqus CAE Manual; Dassault Systemes Simulia Corp: Providence, RI, USA, 2014. 